\title{
The autonomous innervation of the buffalo (Bubalus bubalis) testis. An immunohistochemical study
}

\author{
P. de Girolamo*, C. Costagliola, C. Lucini, G. Gargiulo, L. Castaldo \\ Dipartimento di Strutture, Funzioni e Tecnologie Biologiche; Università di Napoli "Federico II", Naples, \\ Italy
}

C2003, European Journal of Histochemistry

The innervation pattern in the buffalo testis was determined by using histochemical and immunohistochemical methods. Nerves were concentrated in the tunica albuginea and septula testis, and did not show an uniform distribution. The tunica albuginea at the lateral and medial sides and at the free border of the testis is most densely innervated than at the epididymal border. At the cranial pole thick nerve bundles were observed between albugineal vessels and muscle bundles. Rare parenchymal nerves were found in perivascular position between seminiferous tubules and their occurrence is confined to lobules at the cranial and caudal testicular poles. An intense NPY immunoreactivity occurred in nerve bundles and in solitary varicose fibres. Nerves were concentrated in the tunica albuginea at the lateral and medial side and at the free border of the testis, and in the lobules at the cranial and caudal testicular poles. Sub P immunoreactivity was occasionally detected in some thicker nerve bundles and solitary fibers, in the tunica albuginea and in the wall of blood vessels, showing a similar distribution but less intensity and density than NPY immunoreactivity. TH immunoreactivity stained nerve fibers in the buffalo testis with a distribution pattern similar to that obtained with general neuronal markers. The histochemical reaction for AchE was negative, so cholinergic fibers cannot be detected in the buffalo testis. The histochemical NADPHd reaction stained rare nitrergic nerve bundles and solitary fibers. The majority of NADPHd activity was confined to the vascular endothelium, and rarely to the interstitial Leydig cells, whereas the Sertoli and germ cells did not show any reaction.

Key words: autonomous innervation, testis, buffalo, immunohistochemistry.

Correspondence: Paolo de Girolamo, Dipartimento di Strutture, Funzioni e Tecnologie Biologiche, Università di Napoli “Federico II", via Veterinaria 1, 80137 Naples, Italy.

Phone: international +39.081.5644204 Fax: international +39.081.56230. E-mail: degirola@unina.it

Paper accepted on November 15, 2002.

European Journal of Histochemistry 2003; vol. 47 issue 2 [Apr-Jun]:159-164
T he autonomic nervous system has an important role in the regulation of the mammalian male reproductive functions (for review, see Dail 1993). It is a general acknowledge that the intrinsic testicular innervation is mainly associated with blood vessels and involved in the control of testicular blood flow and temperature (Hodson, 1970). In species with smooth muscle in the testicular tunica albuginea and visceral serosa the sympathetic nervous system may participate in the extrusion of spermatozoa from the testis (Davies and Langford, 1971; Bell and McLean, 1973).

The organization and the chemical coding of testicular nerve fibers of mammals have been the subject of many morphological and physiological studies, mostly focused on laboratory mammals (Greenberg et al., 1985; Campos et al., 1990; Properzi et al., 1992) and man (Vaalasti et al., 1986; Taino, 1994). Other mammalian species including large, breeding mammals have largerly been ignored, altough there is some evidence suggesting the existence of distinct species-dependent variations in the neurochemical coding of nerve fibers innervating the testis (Wrobel and Abu-Ghali, 1997; Wrobel and Brandl, 1998; Wrobel and Moustafa, 2000). Particularly, in the testis of bull, pig and donkey local differences in the innervation quality and quantity have been reported. The porcine testis was completely devoid of intrinsic innervation in adult subject, while displays evident age-related changes (Wrobel and Brandl, 1998). No cholinergic and myelinated fibers have been detected in this species, and NPY was the dominating neuropeptide in the testis (Wrobel and Brandl, 1998). The testicular innervation pattern in the donkey is not uniform, is avoid of cholinergic fibers, and shows NPY as the most frequent peptidergic transmitter (Wrobel and KamelMoustafa, 2000). In bovine testis there was no evidence for a cholinergic innervation, and about half of the testicular nerves are NPY immuno-positive (Wrobel and Abu-Ghali, 1997). 
The water buffalo (Bubalus bubalis) is a domesticated ruminant species of considerable economic interest, for both meat and milk production. This species exibits seasonal sexual activity, and is known for its poor reproductive rate differently to bovine. Since there is only one paper, to our knowledge, regarding the structure and innervation of seminal vescicle in water buffalo (Abou Elmagd et al., 1992), further informations on morphology and functions of its genital organs are highly desirable.

Thus, the present study was aimed at investigating the distribution and immunohistochemical properties of intrinsic innervation in the buffalo testis.

\section{Materials and Methods \\ Sampling, fixation, sectioning}

The present investigation was performed on fifteen testes from mature water buffalo (Bubalus bubalis). Samples of testis taken from different regions were immediately removed and fixed by vascular perfusion of Bouin's fluid or $4 \%$ paraformaldehyde, and postfixed by immersion in the same fixatives. Then, same samples were dehydrated in an ethanol series, embedded in paraffin wax, and serially cut into longitudinal and horizontal sections 6-8 $\mu \mathrm{m}$ thick; other samples were washed in 0.1 PBS, trasferred into a graded series of saccarose $(10 \%, 20 \%, 30 \%)$, immersed in Tissue teck OCT compound, snap frozen in liquid nitrogen, and finally sectioned by cryostat, mounted on gelatin/chrome alum-coated slides and air dried.

\section{Immunochemistry}

Simple immunohistochemical staining was performed using the peroxidase anti-peroxidase (PAP) method according to Sternberger (1986). Endogenous peroxidase activity was blocked by treating sections with $3 \%$ hydrogen peroxide for $20 \mathrm{~min}$ at room temperature (RT).

Than the section, rinsed for $15 \mathrm{~min}$ in $0.01 \mathrm{M}$ phosphate buffered saline (PBS) at $\mathrm{pH} 7.4$, were incubated with normal goat serum diluted 1:5 (Jackson Immunoresearch Laboratories, Inc; West Grove, PA, USA; 005-000-121) for $30 \mathrm{~min}$ at RT to prevent background and successively incubated overnight at $4^{\circ} \mathrm{C}$ with polyclonal primary antisera (see Table 1). The section were then rinsed in PBS for $15 \mathrm{~min}$ and incubated with goat-anti rabbit IgG (1:50; Vector Lab, Burlingame, CA, USA; AI-1000) for $30 \mathrm{~min}$ at RT. Subsequently, they were rinsed in PBS for 15 min and incubated with PAP complex (1:100; UCB, Braine-I'Alleud, Belgium) for 30 min at RT. The peroxidase reaction was visualized with a solution of $3.3^{\prime}$ diaminobenzidine tetrahydrocloride (DAB; Sigma; D 5905; $10 \mathrm{mg}$ in $15 \mathrm{~mL}$ of $0.5 \mathrm{M}$ Tris buffer $\mathrm{pH}$ 7.6, containing $1.5 \mathrm{~mL}$ hydrogen peroxide at $0.03 \%$ ). Each incubation was performed in a moist chamber. Finally, the sections counterstained with hematoxylin were dehydrated through an ethanol series, cleared in Xilene, mounted, observed and photographed using a Leitz Aristoplan light microscope. For fluorescence microscopy, the dewaxed sections were rinsed in PBS for 15 min,

Table 1: Details of primary antibodies used in the study.

\begin{tabular}{|c|c|c|c|c|c|}
\hline ANTISERA & ANTIGEN & SOURCE & SPECIFICITY & DILUTION & HOST \\
\hline Protein Gene Product 9.5 (PGP 9.5) & Human brain & Biogenesis & No cross reaction & $1 / 16000$ & rabbit \\
\hline Neurofilament Protein (NF) & $\mathrm{NF} 68 \mathrm{kD}$ & Biogenesis & Cross reaction less than $2 \%$ with the $150 \mathrm{kD}$ or $200 \mathrm{kD}$ forms & $1 / 500$ & rabbit \\
\hline Calcitonine Gene Related Peptide (CGRP) & Rat CGRP & Peninsula IHC 6006 & Cross reaction $10 \%$ with CGRP II no cross reaction with rat amylin & $1 / 500$ & rabbit \\
\hline Cholecystokinin (CCK) & CCK 26-33 & Peninsula IHC 7181 & Cross reaction with Gastrin & $1 / 500$ & Rabbit \\
\hline Neuron Specific Enolase (NSE) & Human brain & Dakopatts & Cross reaction with the isoenzyme of NSE which contain $\gamma$-subunits & $1 / 200$ & Rabbit \\
\hline Neuropeptide Y (NPY) & Porcine NPY & Peninsula IHC 7172 & Cross reaction with PYY & $1 / 2000$ & Rabbit \\
\hline Galanin (Gal) & Porcine Galanin & UCB & No cross reaction with NPY, CCK, Sub P, VIP, NT. & $1 / 2000$ & Rabbit \\
\hline Met- Enkephalin (Menk) & Human Met-Enk & UCB i672/002 & No cross reaction & $1 / 300$ & Rabbit \\
\hline Leu-Enkephalin (Lenk) & Leu5-enkephalin & UCB i $671 / 002$ & No cross reaction & $1 / 500$ & Rabbit \\
\hline Vasoactive Intestinal Polypeptide (VIP) & Porcine VIP & Incstar 20077 & Slight cross reaction with GHRF, PHI and PP. & $1 / 1500$ & Rabbit \\
\hline Substance P (SubP) & Human SubP & Incstar 20064 & No cross reaction & $1 / 2000$ & Rabbit \\
\hline Tyrosine-Hydroxylase (TH) & Rat pheochromocytoma & Chemicon AB 152 & No cross reaction & $1 / 500$ & Rabbit \\
\hline
\end{tabular}


incubated with normal donkey serum (1:5; Jackson Immunoresearch Laboratories; 017-000-121) for 30 min at RT and then incubated overnight at $4^{\circ} \mathrm{C}$ in a moist chamber with polyclonal primary antisera. The sections were then rinsed in PBS for 15 min and incubated with donkey-anti rabbit IgG conjugated to fluorescein isothiocyanate (FITC; 1:25; Jackson Immunoresearch Laboratories; 711-095-152) for 1 $h$ at RT. Fluorochrome-stained sections were rinsed in three changes of PBS of 5 min each, mounted in phosphate-buffered glycerine (9:1) and examined with a Leitz Aristoplan microscope equipped for epifluorescence.

The specificity of the immunoreactivity (IR) was tested by successively substituting PBS for the primary antisera, or the anti-rabbit igG, or the PAP complex, in repeated trials. The cross-reactivity was tested by incubating the sections with antibodies that had been preincubated with excessive amounts of their homologous (up to $50 \mu \mathrm{g} / \mathrm{mL}$ antiserum in the final dilution) and heterologous (up to $100 \mu \mathrm{g} / \mathrm{mL}$ antiserum in the final dilution) antigens.

\section{Histochemistry}

For acetylcholinesterase (AChE) histochemistry the modified, direct-coloring method of Kujat et al. (1993) was employed. Following a short incubation (1-2h) this method allows the specific visualization of cholinergic nerves, while following an incubation time of $25 \mathrm{~h}$ (long incubation) the method reliably illustrates the general autonomous innervation pattern as completely as suitable pan-neuronal immunohistochemical markers.

NADPH diaphorase activity was localized by incubation with $0.25 \mathrm{mg} / \mathrm{mL}$ nitroblue-tetrazolium, 1 $\mathrm{mg} / \mathrm{ml} \beta \mathrm{NADPH}$, and $0.5 \%$ triton $\mathrm{X}-100$ in $0.1 \mathrm{M}$ TRIS- $\mathrm{HCl}$ buffer at $37^{\circ} \mathrm{C}$ for $10-15$ min in a dark box. The reaction was stopped by immersion in TRIS$\mathrm{HCl}$. Control sections included incubation in media in which the substrate was omitted and preincubation in the sulphydryl inhibitor, 5,5'-dithio-bis-(2-nitrobenzoic acid).

\section{Results}

The innervation pattern in the buffalo testis was determined by using histochemical and immunohistochemical methods and comparing a number of general neuronal markers: immunoreactivity for PGP 9.5, NSE, NF and the AchE technique (long incubation). Immunoreactions with antisera to $\mathrm{NF}$ and PGP 9.5 clearly depicted the overall nerve distribution. The immunostaining were equally strong in larger nerve bundles (Figure $1 \mathrm{~A}, \mathrm{~B}$ ) as in the finest ramifications. However, PGP 9.5 also reacts with non-nervous structures such as spermatogenic cells in the tubular compartment of the testis, a fraction of the Leidyg cells and the vascular endothelia. Immunostaining with antiserum to NSE often failed to show smaller and single nerve fibers. AChE reaction (long incubation) demonstrated the general autonomous innervation pattern similar to PGP 9.5 and NF immunohistochemistry.

Nerves were concentrated in the tunica albuginea and septula testis, and did not show an uniform distribution (Figure 4). The tunica albuginea at the lateral and medial sides and at the free border of the testis is most densely innervated than at the epididymal border. At the cranial pole thick nerve bundles were observed between albugineal vessels and muscle bundles. Rare parenchymal nerves were found in perivascular position between seminiferous tubules and their occurrence is confined to lobules at the cranial and caudal testicular poles.

The occurrence of peptidergic nerve fibers has been studied with antibodies to NPY, Gal, Sub P, Met- and Leu-Enk, VIP, CGRP, CCK. NPY occurred in intense immunopositive nerve bundles (Figure 1C), in solitary varicose fibres (Figure 2A), and surronding blood vessels. The highest density observed was similar to that observed with general markers. Nerves were concentrated in the tunica albuginea at the lateral and medial side and at the free border of the testis, and in the lobules at the cranial and caudal testicular poles.

Sub P immunoreactivity was occasionally detected in some thicker nerve bundles and solitary fibers, in the tunica albuginea (Figure 2B) and in the wall of blood vessels, showing a similar distribution but less intensity and density than NPY immunoreactivity.

The immunohistochemical reactions for the determination of CGRP, Met- and Leu-Enk, VIP, and Galanine were totally negative. $\mathrm{TH}$ immunoreactivity stained nerve fibers in the buffalo testis with a distribution pattern similar to that obtained with general neuronal markers (Figure 3A, B). The histochemical reaction for AchE (short incubation) was negative, so cholinergic fibers cannot be detected in the buffalo testis.

The histochemical NADPHd reaction stained rare nitrergic nerve bundles and solitary fibers. The majority of NADPHd activity was confined to the vascular endothelium, and rarely to the interstitial Leydig cells, whereas the Sertoli and germ cells did not show any reaction. 


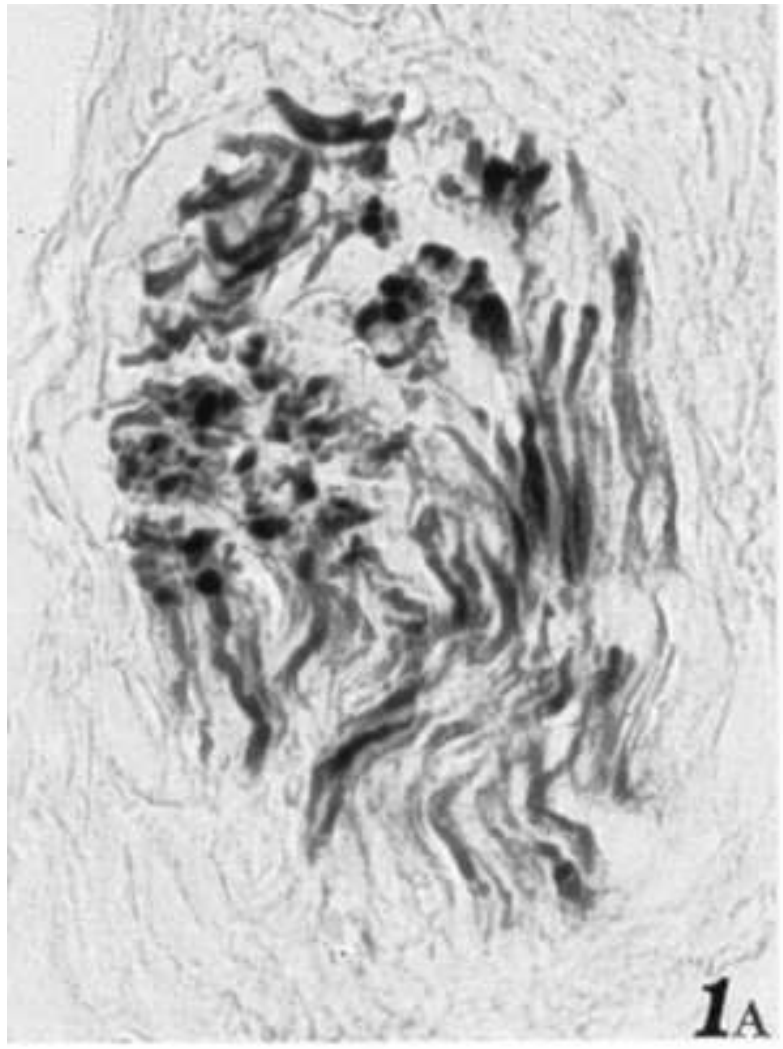

Figure 1: A, B, C: Bright-field micrographs showing immunoreactive large nerve bundles in the tunica albuginea at the medial testicular side. (A) NF (x1100); (B) PgP 9.5 (x1000); (C) NPY (x1800).

\section{Discussion}

In the present study the distribution pattern of the testicular nerves and their chemical coding were demonstrated using histochemical and immunohistochemical methods in the buffalo testis.

Similarly to other large ungulates, the distribution pattern of the testicular nerves showed conspicuous local variations in the buffalo testis. Nerves are concentrated in the tunica albuginea and septula testis, and rare parenchymal nerves were distributed in perivascular portion between seminiferous tubules at the cranial and caudal testicular poles. In the adult
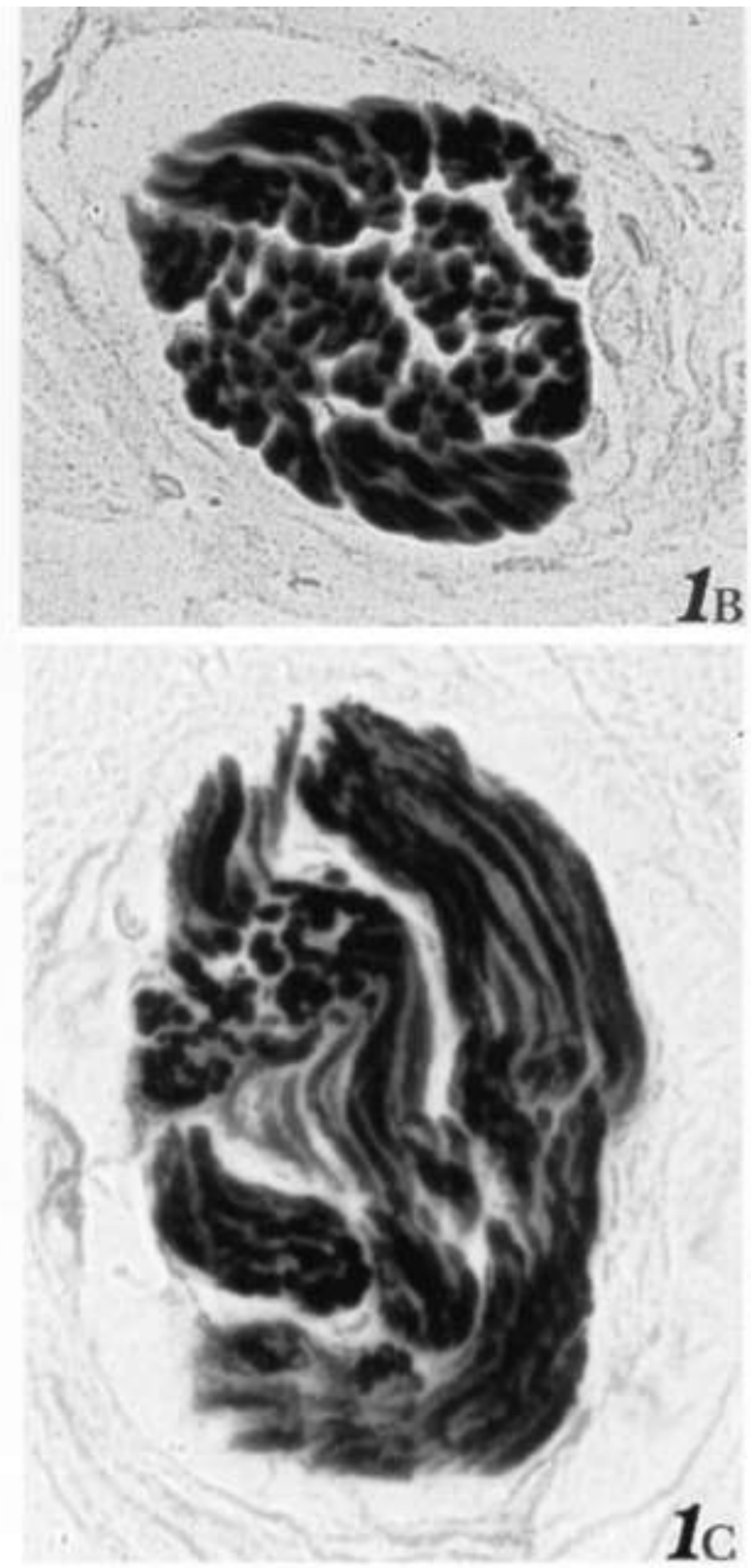

bull the large vessels of the tunica albuginea display a discontinous innervation: a vascular plexus is present in certain areas whereas other portions of the albugineal vascular tree are devoid of intrinsic nerves (Wrobel and Abu-Ghali 1997). The caudal half of the adult bovine testis is completely free of stromal, mediastinal and parenchymal nerves, and in the cranial quarter vascular nerves accompany the large centripetal arteries between the testicular lobes (Wrobel and Abu-Ghali 1997). In the adult donkey the tunica albuginea at the lateral and medial sides and at the free border of the testis is signif- 

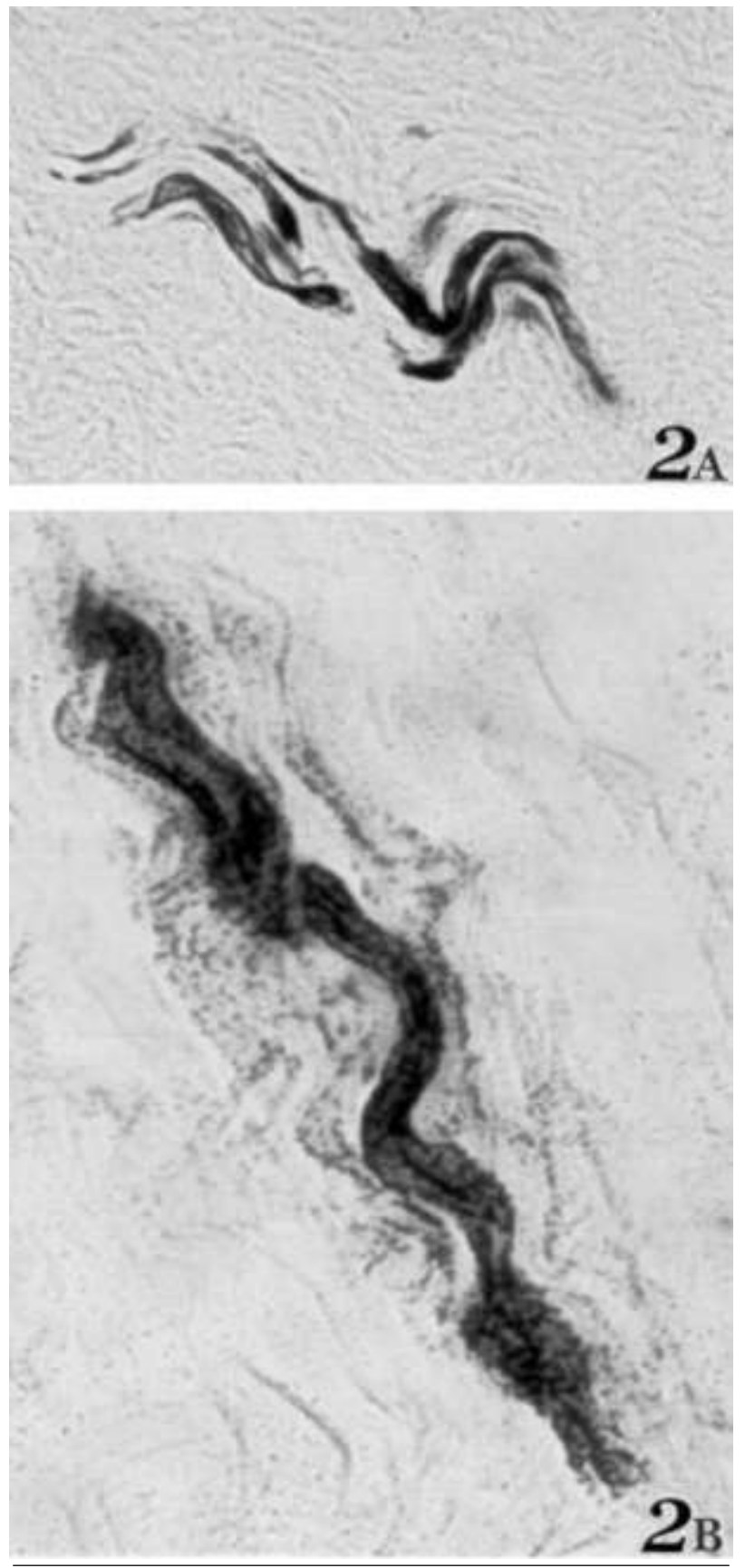

Figure 2 A, B: Bright-fields micrographs showing immunoreactive nerve bundles in the tunica albuginea at the lateral testicular side. (A) NPY (x1100); (B) Sub. P (x1600). icantly less densely innervated than at the epididymal side and the testicular poles (Wrobel and Moustafa, 2000). In the adult pig testis is not completely innervated, and nerve fibers supply the vascular structures of the tunica albuginea and nearly all the septula testis and the mediastinum (Wrobel and Brandl,
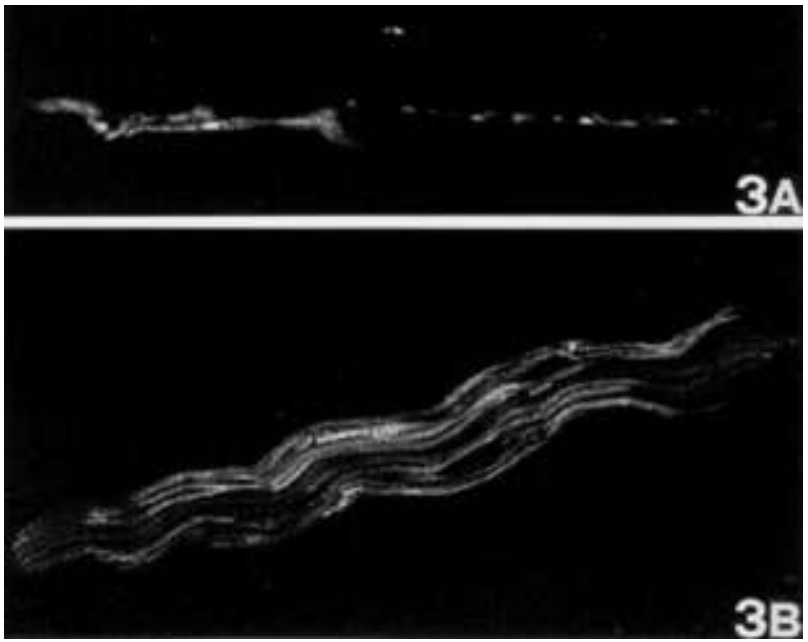

Figure 3 A, B: Fluorescence micrographs showing TH immunopositive nerve fibers in the tunica albuginea at the lateral $(A)$ and medial (B) testicular side. (A: x960; B: x820).

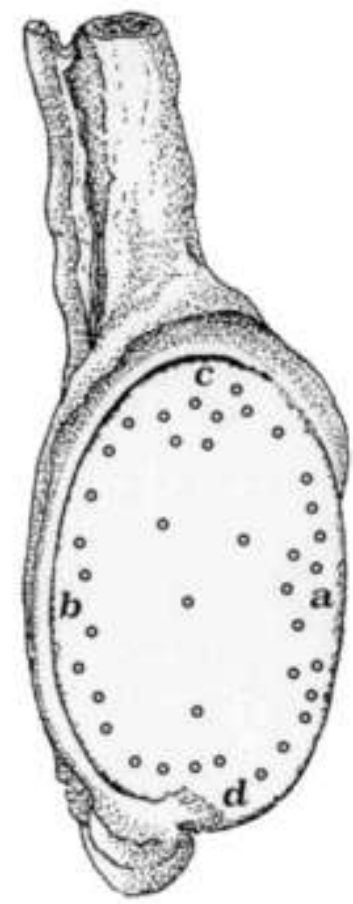

Figure 4. Diagram representing nerve density in the water buffalo testis. Caudal view of the right testis. a: free border; b: epididymal border; c: cranial pole; d: caudal pole. Cranial and caudal poles and the free and epididymal border (tunica albuginea and adjacent parenchyma) of the gonad are well innervated.

1998). Local variations in the density of testicular innervation were detected also in the testis of the toad, in which small fibres bundles as well as single nerve fibres were observed running close to blood vessels or indipendently in the intertubular tissue (Achi et al. 1995). 
Our results on the peptidergic innervation of the buffalo testis shared only partially characteristics described for other mammals. In mammals there are some studies which altogether show marked species differences in the peptidergic innervation of the testis. In the rat testis the innervation appears to be scarce (Zhu et al 1995) or absent (Alm et al, 1980; Properzi et al 1992), while in the guinea pig a rather dense Sub $P$ and VIP-IR innervation has been described (Alm 1980; Larsson 1977). Similar to our results, NPY is the dominating neuropeptide also in the male gonad of the bovine, pig and donkey (Wrobel and Abu-Ghali 1997; Wrobel and Brandl 1998; Wrobel and Moustafa 2000). On the contrary, NPY immunoreactivity is rather frequent in the nerves forming the vascular plexuses of testicular, albugineal and septal arteries, but not so frequent in the mediastinum and the testicular lobules of the cat (Wrobel and Gürtler 2001). NPY has been shown to stimulate vasoconstriction either directely or by potentiating responses to noradrenaline (Lundberg et al. 1982; Edvinson et al. 1983). With a modified AchE histochemical technique (Kujat et al. 1993) using a short incubation period ( $2 \mathrm{~h}$ ), no cholinergic fibres were detected in the buffalo testis. These results agree with those obtained in the testis of bull (Wrobel and Abu-Ghali 1997), boar (Wrobel and Brandl 1998), and donkey (Wrobel and Moustafa 2000). In contrast to the findings obtained in these four ungulates, Wrobel and Gürtler (2001) with the same histochemical method demonstrated a large amount of cholinergic nerve fibres in the testis of the cat. Furthermore, the absence of VIPimmunopositive fibres in the buffalo testis, in agree with the same results obtained in the human (Vaalasti et al. 1986) and bull (Wrobel and Abu-Ghali 1998), is a further indication for the absence of a parasympathetic innervation of the buffalo testis since cholinergic neurons are generally VIP-positive (Dail et al. 1990).

In conclusion, the present study showed a testicular nerve pattern distribution in the buffalo male gonad similar to the bull. Furthermore, the existence of a peptidergic innervation composed of two population of fibers containing NPY and Substance $P$ support the concept of neural regulation of gonadal functions in this species, although the ultimate functional role of these nerve fibres remain to be determined.

\section{References}

Abou-Elmagd A, Kujat R, and Wrobel KH. Ultrastructure and innervation of Water Buffalo (Bubalus bubalis) seminal vesicle. Acta Anat 1992; 145:420-9.

Achi MV, Figueroa JM, González Nicolini V, Villar MJ, Tramezzani JH. NPY- and CGRP-like immunoreactive nerve fibers in the testis and mesorchium of the toad (Bufo arenarum). Cell Tissue Res 1995; 281:375-8

Alm P, Alumets J, Hakanson R, Owman C, Sjöberg N0, Stjiernquist M, et al. Origin and distribution of VIP (Vasoactive Intestinal Polypeptide)nerves in the genitourinary tract. Cell Tissue Res 1980;205:337-47.

Bell C, Mc Lean JR. The autonomic innervation of the rat testicular capsula. J Reprod Fertil 1973;32:253-8.

Campos MB, Vitale ML, Calandra RS, Chiocchio SR. Serotonergic innervation of the rat testis. J. Reprod Fert 1998;88:475-9.

Dail WG, Carrillo Y, Walton G. Innervation of the anococcygeus muscle of the rat. Cell. Tissue Res 1990;259:139-46.

Dail WG. Autonomic innervation of male genitalia. In: Nervous control of the urogenital system. Maggi CA, Ed. Harwood, Chur: Switzerland; 1993. p. 69-102.

Davies JR, Langford GA. Comparative responses of the isolated testicular capsule and parenchyma to autonomic drugs. J Reprod Fertil 1971;26:241-5.

Edvison L, Emson P, McCulloch J, TatemotoK, Uddman R. Neuropeptide Y. Cerebrovascular innervation and vasomotor effects in the cat. Neurosci Lett 1983;43:79-84.

Greenberg J, Forssmann WG, Gorgas K. Morphology and innervation of a testicular 'rete mirabile' in the guinea-pig. Anat. Embryol 1985; 173:225-35.

Hodson N. The nerves of the testis, epididymis and scrotum. In: The testis. vol. I, Johnson AD, Gomes WR, Van Denmark NL, Eds. Academic Press: New York; 1970. p. 47-100.

Kujat R, Rose C, Wrobel KH. The innervation of the bovine ductus deferens: comparison of a modified acetylcholinesterase-reactio with immunoreactivities of cholinacetyltransferase and panneuronal markers. Histochemistry 1993;99:231-9.

Larsson LI, Fahrenkrug J, Schaffalitzky de Muckadell OB. Occurence of nerves containing vasoactive intestinal polypeptide immunoreactivity in the male genital tract. Life Sciences 1977;21:503-8.

Lundberg JM, Terenius L, Hökfelt T, Martling CR, Tatemoto K, Mutt V, et al. Neuropeptide $Y$ (NPY)-like immunoreactivity in peripheral noradrenergic neurons and effects of NPY on sympathetic function. Acta Physiol Scand 1982;116:477-80.

Properzi G, Cordeschi G, Francavilla S. Postnatal development and distribution of peptide-containing nerves in the genital system of the male rat. Histochemistry 1992;97:61-8.

Sternberger LA. Immunocytochemistry. John Wiley \& Sons, $2^{\text {nd }}$ Edition: New York; 1986.

Vaalasti A, Tainio H, Pelto-Huikko M, Hervonen A. Light and electron microscope demostation of VIP- and enkephalin-immunoreactive nerves in the human male genitourinary tract. Anat Rec 1986;215:217.

Taino $\mathrm{H}$. Peptidergic innervation of the human testis and epididymis. Acta Histochem 1994;96:415-20.

Wrobel $\mathrm{KH}$, Gurtler A. The nerve distribution in the testis of the cat. Ann Anat 2001;183:297-308.

Wrobel $\mathrm{KH}$, Abu-Ghali N. Autonomic innervation of the bovine testis. Acta Anat 1997;160:1-14.

Wrobel $\mathrm{KH}$, Brand $\mathrm{B}$. The autonomic innervation of the porcine testis in the period from birth to adulthood. Ann Anat 1998;180:145-56.

Wrobel $\mathrm{KH}$, Kamel-Moustafa MN. On the innervation of the donkey testis. Ann Anat 2000;182:13-22.

Zhu BC, Chiocchio AM, Suburo AM, Tramezzani JH. Monoaminergic and peptidergic contributions of the superior and the inferior spermatic nerves of the innervation of the testis in the rat. J Androl 1995; $16: 248-58$.

\section{Acknowledgements}

The authors wish to thank Ms. Annamaria Zollo for the excellent technical assistance. 